\section{On Rate-Distortion With Mixed Types of Side Information}

Michael Fleming, Member, IEEE, and

Michelle Effros, Senior Member, IEEE

\begin{abstract}
In this correspondence, we consider rate-distortion examples in the presence of side information. For a system with some side information known at both the encoder and decoder, and some known only at the decoder, we evaluate the rate distortion function for both Gaussian and binary sources. While the Gaussian example is a straightforward generalization of the corresponding result by Wyner, the binary example proves more difficult and is solved using a multidimensional optimization approach. Leveraging the insights gained from the binary example, we then solve the more complicated binary Heegard and Berger problem of decoding when side information may be present. The results demonstrate the existence of a new type of successive refinement in which the refinement information is decoded together with side information that is not available for the initial description.
\end{abstract}

Index Terms-Binary source, conditional, Heegard-Berger, multidimensional optimization, successive refinement, Wyner-Ziv.

\section{INTRODUCTION}

The use of side information can improve the rate-distortion performance of data compression codes. We are motivated to study the use of side information by several emerging network source coding applications. For example, consider an environmental remote sensing network with several sensors, each of which takes measurements and transmits them to a central base station, which also makes its own measurements. Environmental variables generally exhibit spatial dependence, and we expect the measurements taken by different sensors to be statistically dependent. In encoding its transmission to the base station, each sensor can consider the measurements taken by the base station as side information available to the base station's decoder. If the system uses multi-hop transmissions, then any measurements relayed by a sensor act as side information available both to that sensor's encoder and the base station's decoder.

Fig. 1(a) shows the system in which side information is available at both the encoder and decoder. The rate-distortion function for this system is known as the conditional rate-distortion function [1], and we refer to this system as the conditional rate-distortion system. Fig. 1(b) shows the Wyner-Ziv system [2], [3], in which side information is available only at the decoder. In this correspondence we combine the two types of side information and study the mixed side information (MSI) system, shown in Fig. 1(c).

The multihop sensor network considered above provides a simple example in which both types of side information are present. Another example comes from a system studied by Heegard and Berger [4] and shown in Fig. 1(d), in which the presence of side information at the decoder is unreliable. The system requires two decoders, one for the case when side information is present (decoder 1) and the other for when

Manuscript received November 15, 2003; revised August 15, 2005. This work is supported in part by the National Science Foundation Award CCR-0220039 and under a Grant from the Lee Center for Advanced Networking at Caltech. The material in this correspondence was presented in part at the IEEE International Symposium on Information Theory, Yokohama, Japan, June/July 2003.

M. Fleming is with the Mitchell Madison Group, New York, NY 1022 USA (e-mail: michael.flemming@motchellmadison.com).

M. Effros is with the Department of Electrical Engineering, MC 136-93, California Institute of Technology, Pasadena, CA 91125 USA (e-mail: effros@ caltech.edu).

Communicated by V. A. Vaishampayan, Associate Editor At Large.

Digital Object Identifier 10.1109/TIT.2006.871059

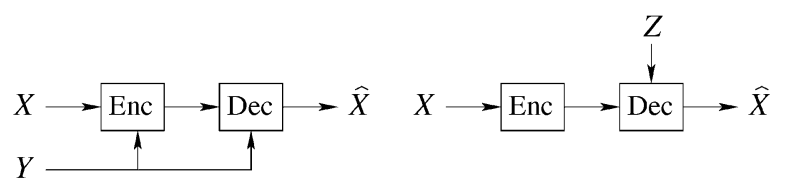

(a)

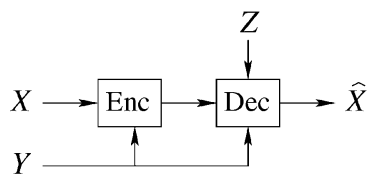

(c) (b)

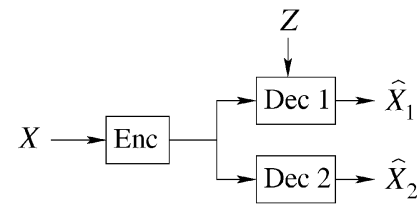

(d)
Fig. 1. (a) The conditional rate-distortion system. (b) The Wyner-Ziv system. (c) The MSI system. (d) Heegard and Berger's system.

it is absent (decoder 2). We can approach coding for this system using a two-part source description. The first part is decoded without side information and ensures a minimum reproduction fidelity at both decoders. The second part requires side information $Z$ for its decoding and serves as refinement information at decoder 1 . It is not useful to decoder 2. Once the first part is chosen, it can be viewed as side information, known to both the encoder and decoder, for the coding of the second part. Thus in coding the second part, we have a mixed side information problem. ${ }^{1}$

In this work, we consider rate-distortion theory for the MSI system. The rate-distortion function for the MSI system follows easily from that for the Wyner-Ziv system. For discrete sources and side information, the rate-distortion function can be obtained directly from a result of Cover and Chiang [6]. The discrete result applies also to continuous sources and side information provided that we impose on the distortion measure the same constraints as were required for the continuous Wyner-Ziv result. We use the MSI rate-distortion function first to generalize Wyner's Gaussian example from the Wyner-Ziv system. We then solve a new binary example that expands significantly on the corresponding example for the Wyner-Ziv system and apply the result to solve the corresponding and more complicated binary example in the Heegard and Berger system.

\section{II. $R(D)$ FOR THE MIXED SidE INFORMATION SySTEM}

In this section, we define notation, derive the rate-distortion function for the MSI system, and bound the system's rate loss.

Let $(\mathcal{X}, \mathcal{Y}, \mathcal{Z}, p(x, y, z))$ be a discrete memoryless 3-source with random variables $X, Y$, and $Z$. We assume $I(X, Y ; Y, Z)<\infty$. Let $\hat{\mathcal{X}}$ be a reconstruction alphabet and let $d: \mathcal{X} \times \hat{\mathcal{X}} \rightarrow[0, \infty)$ be a distortion measure. As in [3], we impose the following two conditions on $d$

1) for all $\hat{x} \in \hat{\mathcal{X}}, \operatorname{Ed}(X, \hat{x})<\infty$;

2) for all random variables $\hat{X}$ such that $0<E d(X, \hat{X})<\infty$, and all $\epsilon>0$, there exists a finite subset $\left\{\hat{x}_{1}, \ldots, \hat{x}_{N}\right\} \subseteq \hat{\mathcal{X}}$, and a quantizer $f_{Q}: \hat{\mathcal{X}} \rightarrow\left\{\hat{X}_{i}\right\}$ such that

$$
\operatorname{Ed}\left(X, f_{Q}(\hat{X})\right) \leq(1+\epsilon) E d(X, \hat{X}) .
$$

Condition 2) is a smoothness constraint used in generalizing the Wyner-Ziv rate-distortion proof from discrete to continuous alphabets

\footnotetext{
${ }^{1}$ The work in this correspondence was initially motivated by a desire to close the gap in the bounds on the binary-source rate-distortion example proposed by Heegard and Berger in [4] and considered further in [5]. In this work we provide a solution that does indeed close that gap, but it has a numerical focus and is not easily characterized analytically.
} 
[3]. Wyner notes that it is not especially restrictive, showing that when $\mathcal{X}=\mathbb{R}$ it holds for all $r$ th power distortion measures, $d(x, \hat{x})=$ $|x-\hat{x}|^{r}$ with $r>0$.

The MSI rate-distortion function, ${ }^{2} R_{X \mid Y\{Z\}}(p, D)$, is given by Lemma 1.

Lemma 1:

$$
\begin{aligned}
R_{X \mid Y\{Z\}}(p, D) & =\underset{W \in \mathcal{M}_{X \mid Y\{Z\}}(p, D)}{\inf _{W}} I(X ; W \mid Y, Z) \\
& =\underset{W \in \mathcal{M}_{X \mid Y\{Z\}}(p, D)}{\inf }[I(X ; W \mid Y)-I(W ; Z \mid Y)],
\end{aligned}
$$

where $\mathcal{M}_{X \mid Y\{Z\}}(p, D)$ is the set of all random variables $W$ described by a test channel $\mu(w \mid x, y)$ with the property $W \rightarrow(X, Y) \rightarrow Z$ and for which there exists an $f: \mathcal{W} \times \mathcal{Y} \times \mathcal{Z} \rightarrow \hat{\mathcal{X}}$ such that

$$
\iiint \int p(x, y, z) \mu(w \mid x, y) d(x, f(w, y, z)) d w d x d y d z \leq D
$$

If the alphabets $\mathcal{X}, \mathcal{Y}$, and $\mathcal{Z}$ are finite, then the infimum becomes a minimum, and it suffices to consider in that minimum only those $W$ with $|\mathcal{W}| \leq|\mathcal{X}||\mathcal{Y}|+1$

For discrete sources, the lemma follows from the result of Cover and Chiang [6] by setting their side information $S_{1}$ at the encoder to be $Y$, and their side information at the decoder to be $(Y, Z)$. For continuous sources, it is obtained in a straightforward way from the Wyner-Ziv rate-distortion function by adopting an approach described by Merhav and Shamai in a comment at the end of [7, Sec. II]: set the source in the Wyner-Ziv problem to be $(X, Y)$, the side information at the decoder to be $(Y, Z)$, and define the distortion measure so that it measures the distortion only of $X$.

We can decompose the rate-distortion function over different values of the side information $Y$ using the following theorem. The proof parallels Gray's proof for the discrete conditional rate-distortion function [8] and is omitted.

Theorem 1: Let $R_{X \mid y\{Z\}}(p, D)$ denote the rate-distortion function for the MSI system when $Y=y$ is constant. Then

$$
R_{X \mid Y\{Z\}}(p, D)=\inf _{\left\{D_{y}\right\} \in \mathcal{D}(p, D)} \int_{y} R_{X \mid y\{Z\}}\left(p, D_{y}\right) p(y) d y
$$

where

$$
\mathcal{D}(p, D)=\left\{\left\{D_{y}, y \in \mathcal{Y}\right\}: \int_{y} D_{y} p(y) d y \leq D\right\} .
$$

The minimum on the right-hand side is achieved when the $D_{y}$ are chosen so that the rate-distortion functions $R_{X \mid y\{Z\}}\left(p, D_{y}\right), y \in \mathcal{Y}$, all have the same slope at their respective distortions $D_{y}$.

Theorem 1 makes rigorous the intuition that we can code distinctly for each value of $y$ and that the distortion at different $y$ values should differ so that all rate-distortion curves operate at points of equal slope.

Zamir [9] defines the rate loss for the Wyner-Ziv system as the difference between the Wyner-Ziv and the conditional rate-distortion functions, $L_{X \mid\{Z\}}(p, D)=R_{X \mid\{Z\}}(p, D)-R_{X \mid Z}(p, D)$. Using the approach from [9], we find that the same rate loss bound derived by Zamir for the Wyner-Ziv system also applies to the mixed side information system.

${ }^{2}$ Set notation in the subscript denotes side information available only at the decoder. Following this pattern, we write the conditional rate-distortion function as $R_{X \mid Y}(p, D)$ and the Wyner-Ziv rate-distortion function as $R_{X \mid\{Z\}}(p, D)$.

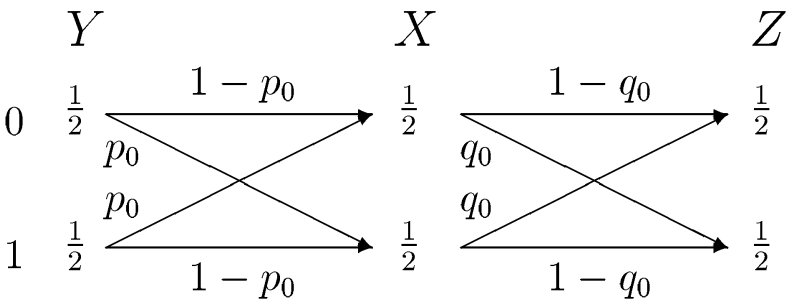

Fig. 2. Joint distribution of $(X, Y, Z)$ for the binary MSI example.

\section{JOINTLY GAUSSIAN SOURCES}

Consider a zero-mean, jointly Gaussian random variable $(X, Y, Z)$ with $E X^{2}=\sigma_{X}^{2}$. Denote by $K$ the covariance matrix of $(X, Y, Z)$, and let $L=\left(l_{i j}\right)=K^{-1}$. Given $Y$ and $Z, X$ is Gaussian with conditional mean and variance given by

$$
\mathrm{E}[X \mid Y, Z]=-\frac{l_{12}}{l_{11}} Y-\frac{l_{13}}{l_{11}} Z, \operatorname{Var}[X \mid Y, Z]=\frac{1}{l_{11}} \text {. }
$$

Following an approach similar to that used by Wyner [3], we obtain

$$
\begin{aligned}
R_{X \mid Y\{Z\}}(p, D) & =R_{X \mid Y Z}(p, D) \\
& = \begin{cases}\frac{1}{2} \log \frac{1}{l_{11} D}, & 0<D<\frac{1}{l_{11}} \\
0, & D \geq \frac{1}{l_{11}} .\end{cases}
\end{aligned}
$$

where $R_{X \mid Y Z}(p, D)$ is the conditional rate distortion function of $X$ given $(Y, Z)$. As in the Wyner-Ziv case, the rate-distortion function is the same whether the side information $Z$ is available at the encoder or not.

\section{JOINTLY BINARY SOURCES}

Let $X, Y$, and $Z$ be binary sources, and let $Y$ and $Z$ be related to $X$ via binary symmetric channels, as shown in Fig. 2. All three variables have marginals of $\left(\frac{1}{2}, \frac{1}{2}\right)$, and we denote the crossover probabilities of the channels by

and

$$
\operatorname{Pr}(X=1 \mid Y=0)=\operatorname{Pr}(X=0 \mid Y=1)=p_{0}<\frac{1}{2}
$$

$$
\operatorname{Pr}(Z=1 \mid X=0)=\operatorname{Pr}(Z=0 \mid X=1)=q_{0}<\frac{1}{2}
$$

as shown. We denote by $p(x, y, z)$ the joint distribution, and we adopt the Hamming distortion measure $(d(x, \hat{x})=0$ if $x=\hat{x}$ and $d(x, \hat{x})=$ 1 if $x \neq \hat{x}$ ).

For this problem, Theorem 1 gives

$$
R_{X \mid Y\{Z\}}(D)=\frac{1}{2} R_{X \mid Y=0\{Z\}}\left(D_{0}\right)+\frac{1}{2} R_{X \mid Y=1\{Z\}}\left(D_{1}\right)
$$

for some $D_{0}$ and $D_{1}$ such that $\frac{1}{2} D_{0}+\frac{1}{2} D_{1}=D$. By symmetry, $D_{1}=D_{2}=D$, and $R_{X \mid Y=0\{Z\}}\left(D_{1}\right)=R_{X \mid Y=1\{Z\}}\left(D_{2}\right)$. Thus,

$$
R_{X \mid Y\{Z\}}(D)=R_{X \mid Y=0\{Z\}}(D)=R_{X \mid\{Z\}}(q, D)
$$

where $q(x, z)=p(x, z \mid y=0)$. From here on, we concentrate on finding $R_{X \mid\{Z\}}(q, D)$. The problem is similar to the binary symmetric example solved in [2], except that the marginals on $X$ and $Z$ are now skewed: the marginal on $X$ is $\left(1-p_{0}, p_{0}\right)$, and that on $Z$ is $\left(1-p_{0} *\right.$ $\left.q_{0}, p_{0} * q_{0}\right)$, where $a * b \triangleq a(1-b)+b(1-a)$.

By the cardinality bound on the auxiliary random variable [2], we can restrict the minimum in the definition of $R_{X \mid\{Z\}}(q, D)$ to consider 
$W$

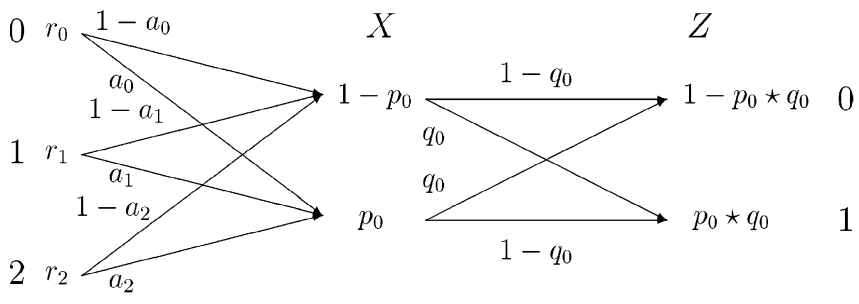

Fig. 3. Joint distribution of $(W, X, Z)$ for $|\mathcal{W}| \leq 3$.

only those $W$ with $|\mathcal{W}| \leq 3$. The general form of such a $W$ is shown in Fig. 3. From that figure, we have

$$
\begin{aligned}
I(X ; W)-I(W ; Z) \\
=H(X)-H(X \mid W)-H(Z)+H(Z \mid W) \\
=H(X)-H(Z) \\
\quad+\sum_{i=0}^{2} \operatorname{Pr}(W=w)[H(Z \mid W=w)-H(X \mid W=w)] \\
=H\left(p_{0}\right)-H\left(p_{0} * q_{0}\right)+\sum_{w=0}^{2} r_{w}\left[H\left(a_{w} * q_{0}\right)-H\left(a_{w}\right)\right] \\
=-G\left(p_{0}\right)+\sum_{w=0}^{2} r_{w} G\left(a_{w}\right)
\end{aligned}
$$

where

$$
G(u) \triangleq H\left(u * q_{0}\right)-H(u)
$$

The parameters $\left(a_{0}, a_{1}, a_{2}, r_{0}, r_{1}, r_{2}\right)$ obey the constraints $0 \leq a_{w} \leq$ $1, r_{w} \geq 0$ for all $w \in\{0,1,2\}$, and also

$$
\begin{aligned}
r_{0}+r_{1}+r_{2} & =1 \\
r_{0} a_{0}+r_{1} a_{1}+r_{2} a_{2} & =p_{0} .
\end{aligned}
$$

Let $\mathcal{F}=\{f: \mathcal{W} \times \mathcal{Z} \rightarrow\{0,1\}:|\mathcal{W}|=3,|\mathcal{Z}|=2\}$. Then we can write

$$
R_{X \mid\{Z\}}(q, D)=\min _{f \in \mathcal{F}} R_{X \mid\{Z\}}(q, D, f)
$$

where we get (4) at the bottom of the page and $\mathcal{M}_{X \mid\{Z\}}(p, D, f)$ is the set of all random variables $W$ with $|\mathcal{W}|=3$ described by a test channel $\mu(w \mid x)$ with the property $W \rightarrow X \rightarrow Z$ and for which

$$
\sum_{w=0}^{2} \sum_{x=0}^{1} \sum_{z=0}^{1} q(x, z) \mu(w \mid x) d(x, f(w, z)) \leq D .
$$

To compute the rate-distortion function, we consider each possible decoder $f \in \mathcal{F}$ in turn, and evaluate the minimization over $W \in \mathcal{M}_{X \mid\{Z\}}(p, D, f)$ for each.
TABLE I

Possible Decoding Functions For EACH Symbol, Together With Their EXPECTED DISTORTION CONTRIBUTION

\begin{tabular}{|c|c||c|}
\hline$f(w, 0)$ & $f(w, 1)$ & $r_{w} q(z \mid w) \operatorname{Pr}(X \neq f(w, z))$ \\
\hline \hline 0 & 0 & $r_{w} a_{w}$ \\
\hline 0 & 1 & $r_{w} q_{0}$ \\
\hline 1 & 0 & $r_{w}\left(1-q_{0}\right)$ \\
\hline 1 & 1 & $r_{w}\left(1-a_{w}\right)$ \\
\hline
\end{tabular}

TABLE II

A Possible Decoding Function $f$ When $|\mathcal{W}|=3$

\begin{tabular}{|c||c|c|}
\hline$w$ & $f(w, 0)$ & $f(w, 1)$ \\
\hline \hline 0 & 0 & 0 \\
\hline 1 & 0 & 0 \\
\hline 2 & 0 & 1 \\
\hline
\end{tabular}

For a particular $f$ and $W$, the expected distortion of the system is given by

$$
\begin{aligned}
E d(X, f(W, Z)) & =\sum_{w=0}^{2} \sum_{z=0}^{1} q(w, z) E[d(X, f(W, Z)) \mid W=w, Z=z] \\
& =\sum_{w=0}^{2} \sum_{z=0}^{1} r_{w} q(z \mid w) \operatorname{Pr}(X \neq f(w, z)) .
\end{aligned}
$$

For each symbol, there are four possible choices for the decoding rule $f(w, \cdot)$. These are shown in Table I, together with $r_{w} q(z \mid w) \operatorname{Pr}(X \neq$ $f(w, z))$, their corresponding contribution to the expected distortion. Since $q_{0}<\frac{1}{2}$, we have $r_{w} q_{0}<r_{w}\left(1-q_{0}\right)$, implying that any decoder with $f(w, 0)=1$ and $f(w, 1)=0$ for some $w$ can never be optimal; for any such decoder we could always lower the expected distortion by setting $f(w, 0)=0$ and $f(w, 1)=1$. Therefore, we need not further consider decoders with $f(w, 0)=1$ and $f(w, 1)=0$ for any $w$. For all other decoders, we write the distortion constraint as a function of $r_{w}$ and $a_{w}$ using Table I. Table II gives an example; the corresponding distortion constraint is $r_{0} a_{0}+r_{1} a_{1}+r_{2} q_{0} \leq D$.

Lemma 2 below shows that for any $f$, the distortion constraint is tight at all points of interest on the $R_{X \mid\{Z\}}(q, D, f)$ curve. Thus, we can restrict our attention to test channels that meet the distortion constraint with equality.

Lemma 2: Consider a finite-alphabet Wyner-Ziv system with source $X$, side information $Z$, and decoding function $f$. Let $D_{\text {max }} \triangleq \min \left\{D: R_{X \mid\{Z\}}(q, D, f)=0\right\}$. Then for all $D \leq D_{\max }$, the minimum over all test channels $\mu(w \mid x) \in \mathcal{M}_{X \mid\{Z\}}(q, D, f)$ in the definition of $R_{X \mid\{Z\}}(q, D, f)$ can be replaced by a minimum over the subset of test channels in $\mathcal{M}_{X \mid\{Z\}}(q, D, f)$ for which

$$
\sum_{w \in \mathcal{W}} \sum_{x \in \mathcal{X}} \sum_{z \in \mathcal{Z}} q(x, z) \mu(w \mid x) d(x, f(w, z))=D .
$$

Proof: For any $D_{1}$ and $D_{2}$ such that $D_{1} \leq D_{2}$

$$
\mathcal{M}_{X \mid\{Z\}}\left(q, D_{1}, f\right) \subseteq \mathcal{M}_{X \mid\{Z\}}\left(q, D_{2}, f\right) .
$$

$$
R_{X \mid\{Z\}}(q, D, f)= \begin{cases}\min _{W \in \mathcal{M}_{X \mid\{Z\}}(q, D, f)}\left[-G\left(p_{0}\right)+\sum_{w=0}^{2} r_{w} G\left(a_{w}\right)\right], & \text { if } \mathcal{M}_{X \mid\{Z\}}(q, D, f) \neq \emptyset \\ \infty, & \text { otherwise }\end{cases}
$$


Combining this with a timesharing argument, we have that $R_{X \mid\{Z\}}(q, D, f)$ is convex in $D$ and that $R_{X \mid\{Z\}}(q, D, f)$ is strictly decreasing for all $D$ such that $R_{X \mid\{Z\}}(q, D, f)>0$. Thus, for any $0<D \leq D_{\max }$ and any $\epsilon>0$ we can write

$$
R_{X \mid\{Z\}}(q, D, f)<R_{X \mid\{Z\}}(q, D-\epsilon, f)
$$

i.e.,

$$
\begin{aligned}
\min _{\mu(w \mid x) \in \mathcal{M}_{X \mid\{Z\}}(q, D, f)} I(X ; W \mid Z) \\
<\min _{\mu(w \mid x) \in \mathcal{M}_{X \mid\{Z\}}(q, D-\epsilon, f)} I(X ; W \mid Z) .
\end{aligned}
$$

Since $\mathcal{M}_{X \mid\{Z\}}(q, D-\epsilon, f) \subseteq \mathcal{M}_{X \mid\{Z\}}(q, D, f)$, the $W$ attaining the minimum on the left hand side must be in $\mathcal{M}_{X \mid\{Z\}}(q, D, f)-$ $\mathcal{M}_{X \mid\{Z\}}(q, D-\epsilon, f)$, and such a $W$ must achieve an expected distortion $D(W)$ satisfying $D-\epsilon<D(W) \leq D$. The result then follows from the fact that $\epsilon$ is arbitrary.

To compute the rate-distortion function, we consider each decoding function $f$ in turn. For the $f$ from Table II, the problem is to minimize

$$
g\left(a_{0}, a_{1}, a_{2}, r_{0}, r_{1}, r_{2}\right) \triangleq r_{0} G\left(a_{0}\right)+r_{1} G\left(a_{1}\right)+r_{2} G\left(a_{2}\right)
$$

over all $\left(a_{0}, a_{1}, a_{2}, r_{0}, r_{1}, r_{2}\right)$ that satisfy

$$
\begin{aligned}
r_{0}+r_{1}+r_{2}-1 & =0 \\
r_{0} a_{0}+r_{1} a_{1}+r_{2} a_{2}-p_{0} & =0 \\
r_{0} a_{0}+r_{1} a_{1}+r_{2} q_{0} & =D \\
r_{w} & \geq 0, \quad w \in\{0,1,2\} \\
0 \leq a_{w} & \leq 1, \quad w \in\{0,1,2\} .
\end{aligned}
$$

Since $G$ is convex [2], the function $g\left(r_{0}, r_{1}, r_{2}, a_{0}, a_{1}, a_{2}\right)$ is convex in each of its parameters. Thus, there can be only one local extreme value, and, if it exists, it is the global minimum. The three equality constraints (6), (7), and (8) allow us to reduce the number of unsolved parameters from six to three. We reduce the search space further using insights obtained by applying Lagrange multipliers to the optimization; details are provided in Appendix A. The resulting numerical solution for $R_{X \mid\{Z\}}(q, D, f)$ leaves at most one free parameter, as in the solution of the binary example given by Wyner and Ziv. After finding $R_{X \mid\{Z\}}(q, D, f)$ for each $f, R_{X \mid\{Z\}}(q, D)$ follows from (3).

We summarize in Fig. 4 the form of the optimal solution for various values of $p_{0}$ and $q_{0}$ when $D=0.1$. The results for other values of $D$ are qualitatively the same, but the region for which $R=0$ grows as $D$ grows.

When both $p_{0}$ and $q_{0}$ are close to $D=0.1$, only two symbols are required. Symbol one conveys "set $\hat{X}=0$," and symbol two conveys "set $\hat{X}$ equal to the maximum likelihood predictor for $X$ given the side information." Symbol two costs little rate to describe and gives an expected distortion $E d(X, \hat{X})=\min \left(p_{0}, q_{0}\right)>D$. Symbol one complements symbol two by allowing us to occasionally describe the (skewed) source at a higher quality. As both $p_{0}$ and $q_{0}$ increase, the distortion constraint becomes tighter, and we soon require a third symbol, "set $\hat{X}=1$." When both $p_{0}$ and $q_{0}$ are large, symbol two drops out of use since a reproduction based on the side information has high expected distortion.

In comparing to earlier work, we note that Wyner and Ziv's solution for a symmetric marginal on $X$ is a special case of the three-symbol solution in which $r_{0}=r_{2}$.

\section{HeEgard AND Berger's System}

In [4], Heegard and Berger pose a binary rate-distortion problem for the system of Fig. 1(d). Choosing $X$ and $Z$ to be symmetric binary sources, they relate the two via a binary symmetric channel of crossover probability $q_{0}$ and derive an upper bound on the rate-distortion function. They conjecture that this bound is tight. In [5], Kerpez shows that their bound is loose and provides new upper and lower bounds. In this section, we use the insights gained from the MSI system to show how to compute the rate-distortion function for this example to a high degree of accuracy using an approach that is numerical and is based on highly constrained, multidimensional optimization.

The rate-distortion function for the Heegard and Berger (HB) system is

$$
R_{\mathrm{HB}}\left(D_{1}, D_{2}\right)=\min _{(U, V) \in \mathcal{M}_{\mathrm{HB}}\left(D_{1}, D_{2}\right)}[I(X ; U)+I(X ; V \mid U, Z)]
$$

where $\mathcal{M}_{H B}\left(D_{1}, D_{2}\right)$ is the set of auxiliary random variables $(U, V)$ such that $(U, V) \rightarrow X \rightarrow Z$ and there exist reproduction functions $\hat{X}_{1}=f_{1}(U, V, Z)$ and $\hat{X}_{2}=f_{2}(U)$ such that $\hat{X}_{1}$ and $\hat{X}_{2}$ satisfy $E d\left(X, \hat{X}_{1}\right) \leq D_{1}$ and $E d\left(X, \hat{X}_{2}\right) \leq D_{2}$ respectively [4]. An alternative form is given by Kaspi in [10].

The condition $(U, V) \rightarrow X \rightarrow Z$ can be rewritten using the following lemma.

Lemma 3: The condition $(U, V) \rightarrow X \rightarrow Z$ is equivalent to the two conditions $U \rightarrow X \rightarrow Z$ and $V \rightarrow(U, X) \rightarrow Z$.

Proof: The result follows from

$$
I(U, V ; Z \mid X)=I(U ; Z \mid X)+I(V ; Z \mid U, X)
$$

and the fact that mutual information is nonnegative.

For any $U$, there exists a $V$ and $f_{1}$ such that $E d\left(X, f_{1}(U, V, Z)\right) \leq$ $D_{1}$ (for instance, $V=X$ and $\left.f_{1}(U, V, Z)=V\right)$. We can therefore rewrite $R_{H B}\left(D_{1}, D_{2}\right)$ with the help of Lemma 3 as

$$
\begin{aligned}
R_{\mathrm{HB}}\left(D_{1}, D_{2}\right)= & \min _{U \in \mathcal{M}_{\mathrm{HB}}^{U}\left(D_{2}\right)}[I(X ; U) \\
& \left.+\min _{V \in \mathcal{M}_{\mathrm{HB}}^{V}\left(U, D_{1}\right)} I(X ; V \mid U, Z)\right]
\end{aligned}
$$

where

$$
\begin{aligned}
& \mathcal{M}_{\mathrm{HB}}^{U}\left(D_{2}\right)=\{U: U \rightarrow X \rightarrow Z, \\
&\left.\exists f_{2} \text { s.t. } E d\left(X, f_{2}(U)\right) \leq D_{2}\right\} \\
& \mathcal{M}_{\mathrm{HB}}^{V}\left(U, D_{1}\right)=\{V: V \rightarrow(U, X) \rightarrow Z, \\
&\left.\quad \exists f_{1} \text { s.t. } \operatorname{Ed}\left(X, f_{1}(U, V, Z)\right) \leq D_{1}\right\} .
\end{aligned}
$$

Noting the equivalence of

$$
\mathcal{M}_{\mathrm{HB}}^{V}\left(U, D_{1}\right) \text { and } \mathcal{M}_{X \mid U\{Z\}}\left(p(u, x, z), D_{1}\right)
$$

we have

$R_{\mathrm{HB}}\left(D_{1}, D_{2}\right)=\min _{U \in \mathcal{M}_{\mathrm{HB}}^{U}\left(D_{2}\right)}\left[I(X ; U)+R_{X \mid U\{Z\}}\left(p(u, x, z), D_{1}\right)\right]$.

For the binary example, this yields

$$
\begin{aligned}
R_{\mathrm{HB}}\left(D_{1}, D_{2}\right)=\min _{U \in \mathcal{M}_{\mathrm{HB}}^{U}\left(D_{2}\right)} \sum_{u \in \mathcal{U}} p(u)[1-H(X \mid U=u) \\
\left.+R_{X \mid U=u\{Z\}}\left(p(u, x, z), D_{1}\right)\right] .
\end{aligned}
$$

The variable $U$ must achieve the distortion constraint at decoder 2 . As shown in [4], its alphabet is bounded according to $|\mathcal{U}| \leq|\mathcal{X}|+2=$ 4. We can therefore represent $U$ by its marginal probabilities $r_{u}$ and transition probabilities $a_{u}=\operatorname{Pr}(X=1 \mid U=u), u \in\{0,1,2,3\}$. These parameters must satisfy

$$
\begin{aligned}
r_{0}+r_{1}+r_{2}+r_{3} & =1 \\
r_{0} a_{0}+r_{1} a_{1}+r_{2} a_{2}+r_{3} a_{3} & =\frac{1}{2} \\
0 & \leq r_{u}, \quad u \in\{0,1,2,3\} \\
0 & \leq a_{u} \leq 1, \quad u \in\{0,1,2,3\} .
\end{aligned}
$$



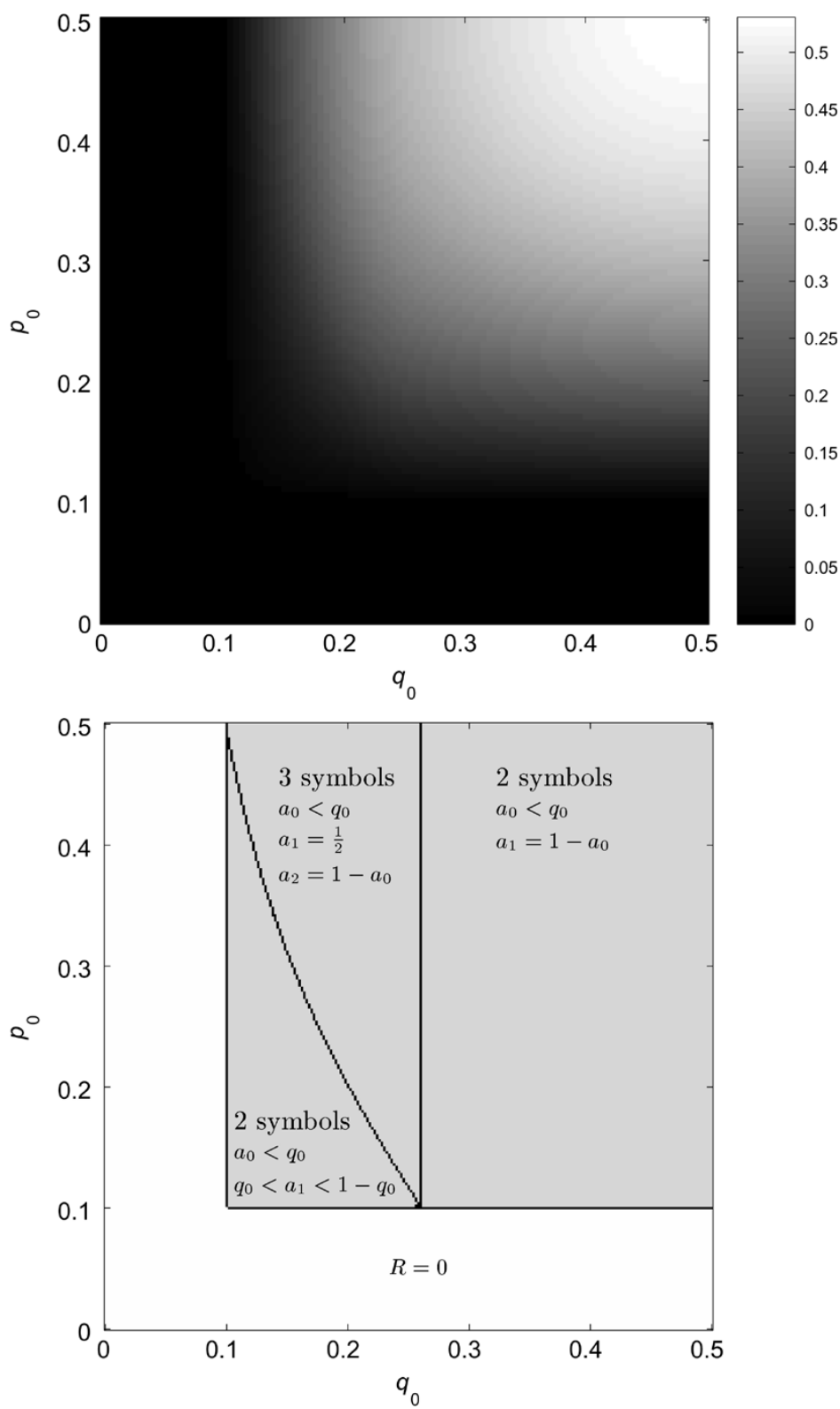

Fig. 4. Value (top) and form (bottom) of the optimal solution for different values of $p_{0}$ and $q_{0}$ when $D=0.1$.

For each $u$, the distribution $p(u, x, z)$ is entirely characterized by that symbol's transition probability $a_{u}$ and the side information crossover probability $q_{0}$. In what follows, we write $R_{X \mid U=u}\{Z\}\left(p(u, x, z), D_{1}\right)$ in the form $R\left(a_{u}, q_{0}, D_{1}\right)$ to make explicit its functional dependence on these parameters. It is the binary MSI rate-distortion function determined in the previous section when $p_{0}=a_{u}$.

Assume that $R\left(a_{u}, q_{0}, D_{1}\right)$ is differentiable ${ }^{3}$ with respect to $a_{u}$, and define $K\left(a_{u}\right) \triangleq R\left(a_{u}, q_{0}, D_{1}\right)-H\left(a_{u}\right)$. Finding the optimal $U$ is equivalent to finding the $\left(a_{0}, a_{1}, a_{2}, a_{3}, r_{0}, r_{1}, r_{2}, r_{3}\right)$ that minimize

$$
1+r_{0} K\left(a_{0}\right)+r_{1} K\left(a_{1}\right)+r_{2} K\left(a_{2}\right)+r_{3} K\left(a_{3}\right)
$$

subject to the constraints (11)-(14) together with a distortion constraint for decoder 2. In Appendix C, we outline how to evaluate this minimization and hence determine $R_{\mathrm{HB}}\left(D_{1}, D_{2}\right)$ using a search over only

${ }^{3}$ We show in Appendix B that although $R\left(a_{u}, q_{0}, D_{1}\right)$ is not differentiable everywhere with respect to $a_{u}$, we can alter it by an insignificant amount so as to smooth it and make it so. two parameters, matching the complexity required to evaluate the existing bounds by Heegard and Berger and Kerpez.

Evaluating $R_{\mathrm{HB}}\left(D_{1}, D_{2}\right)$, we find a significant region of $\left(q_{0}, D_{1}, D_{2}\right)$-space for which the bounds of Heegard and Berger and Kerpez are loose; an example is shown in Fig. 5. We find that the rate-distortion function can at some points be as much as 0.056 bits per symbol below the minimum of the two prior upper bounds, and at others up to 0.2143 bits per symbol above Kerpez's lower bound. ${ }^{4}$

There is one local minimum solution to the minimization that is always present in the case when we assume that none of the inequality constraints is active. That minimum requires two symbols and occurs when $a_{0}=D_{2}, a_{1}=1-D_{2}, r_{0}=\frac{1}{2}$, and $r_{1}=\frac{1}{2}$, i.e., when $U$ is related to $X$ via a binary symmetric channel with crossover probability $D_{2}$. In practice, we find that this is the optimal solution for all $q_{0}, D_{1}$,

${ }^{4}$ We note that, contrary to a conjecture by Kerpez, his solution is not everywhere better than Heegard and Berger's. The authors thank Sidharth Jaggi for verifying a simple counterexample that at $\left(q_{0}, D_{1}, D_{2}\right)=(0.1,0.05,0.25)$, Kerpez's bound $R_{\mathrm{HB}} \leq 0.4116$ is looser than Heegard and Berger's bound $R_{\mathrm{HB}} \leq 0.3970$. 


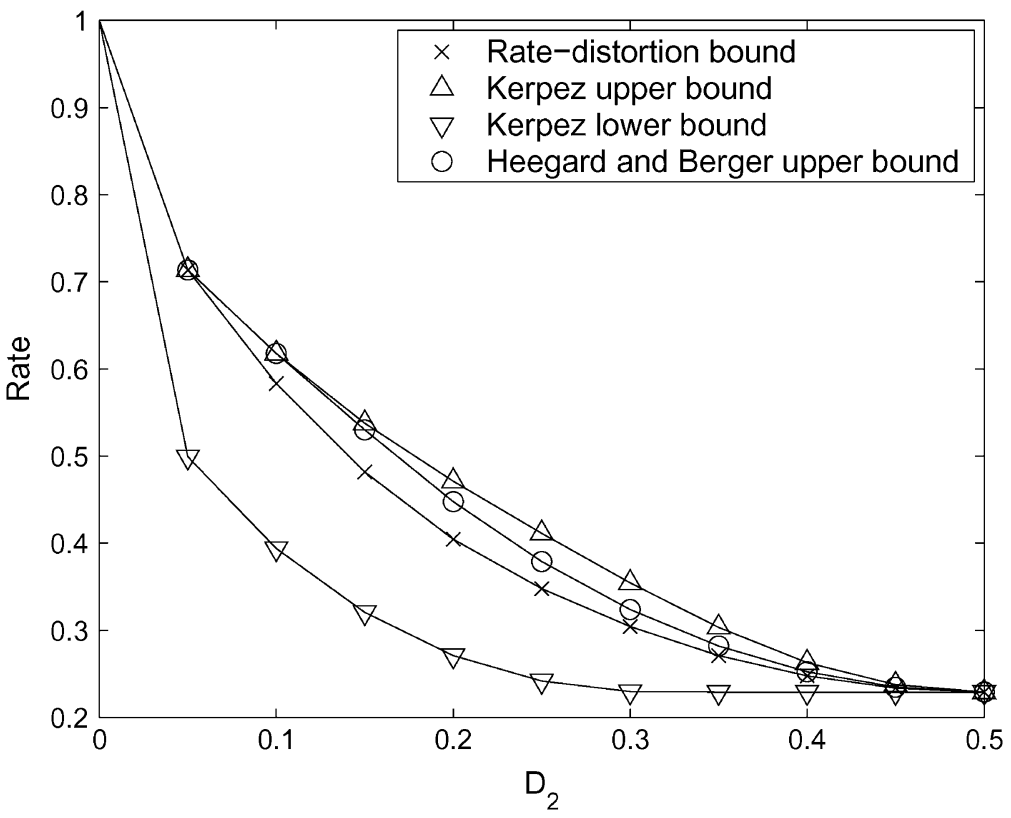

Fig. 5. Numerical results for Heegard and Berger's system, $q_{0}=0.1, D_{1}=0.05$.

and $D_{2}$ tested, and we conjecture that it is a unique optimal solution. However, since $K\left(a_{u}\right)$ is not convex (because $R\left(a_{u}, q_{0}, D_{1}\right)$ is not a convex function of $a_{u}$ ), we cannot easily prove the uniqueness of this solution. We can at least conclude that it provides an extremely tight upper bound, and it can be computed with a search over only one parameter rather than two.

A binary symmetric $U$ achieves the rate-distortion function in the absence of decoder 1 (i.e., $U$ achieves $R_{X}\left(D_{2}\right)$ ). The corresponding $V$ achieves $R_{X \mid U\{Z\}}\left(p, D_{1}\right)$. Here we have a situation akin to successive refinement, except that the refinement variable $V$ must now work in cooperation with side information. Looking at Heegard and Berger's Gaussian example in [4], we find the same pattern. There, too, the variable $U$ is chosen as it would be to achieve $R_{X}\left(D_{2}\right)$, and $V$ is chosen to provide the necessary refinement. We recall that both binary and Gaussian sources are successively refinable, suggesting that this two-step approach might achieve the Heegard and Berger rate-distortion function for all successively refinable sources for which $R_{X}\left(D_{2}\right)$ is achieved by a $U$ generated independent and identically distributed from $X$.

For general sources, the two-step approach bounds the HB rate-distortion function from above in terms of the traditional and MSI rate-distortion functions. The MSI rate-distortion function is in turn bounded in relation to the conditional rate-distortion function by the rate loss results of Section II.

\section{SUMMARY}

We investigate the Gaussian and binary rate-distortion examples for the mixed side information system. The Gaussian example generalizes easily from the Wyner-Ziv case; the binary example is considerably more complicated, but we present an easily computable solution. We use this MSI solution to help us solve a more difficult binary rate-distortion problem for the system of Heegard and Berger. For this system, comparison of the new binary solution and the previously existing Gaussian solution show that they both use a separable, two-step approach to construct auxiliary random variables. That a two-step approach is optimal suggests the existence of a new type of successive refinement for which the second part of the description is decoded together with side information. It also suggests that a two-step approach might yield good results for practical coding.
APPENDIX A

\section{THE BINARY MSI EXAMPLE}

In this Appendix, we apply Lagrange multipliers to the minimization required to determine $R_{X \mid\{Z\}}(q, D, f)$. We use the function $f$ described by Table II to illustrate our method. For this $f$, we seek to minimize (5) subject to the conditions in (6)-(10). There are six inequality constraints; our application of Lagrange multipliers will depend on the subset that is active. Assume first that none of the constraints is active. We use the objective function and the equality constraints to form the Lagrangian

$$
\begin{aligned}
J\left(a_{0}, a_{1}, a_{2}, r_{0}, r_{1}, r_{2}\right)= & r_{0} G\left(a_{0}\right)+r_{1} G\left(a_{1}\right)+r_{2} G\left(a_{2}\right) \\
& +\lambda_{1}\left(r_{0}+r_{1}+r_{2}-1\right) \\
& +\lambda_{2}\left(r_{0} a_{0}+r_{1} a_{1}+r_{2} a_{2}-p_{0}\right) \\
& +\lambda_{3}\left(r_{0} a_{0}+r_{1} a_{1}+r_{2} q_{0}-D\right)
\end{aligned}
$$

and obtain the first-order optimality conditions by differentiating $J\left(a_{0}, a_{1}, a_{2}, r_{0}, r_{1}, r_{2}\right)$ :

$$
\begin{array}{rlrl}
\frac{\partial}{\partial r_{0}}: & & G\left(a_{0}\right)=\lambda_{1}+a_{0} \lambda_{2}+\lambda_{3} \\
\frac{\partial}{\partial r_{1}}: & & G\left(a_{1}\right)=\lambda_{1}+a_{1} \lambda_{2}+\lambda_{3} \\
\frac{\partial}{\partial r_{2}}: & & G\left(a_{2}\right)=\lambda_{1}+a_{2} \lambda_{2}+q_{0} \lambda_{3} \\
\frac{\partial}{\partial a_{0}}: & G^{\prime}\left(a_{0}\right)=\lambda_{2}+\lambda_{3} \\
\frac{\partial}{\partial a_{1}}: & G^{\prime}\left(a_{1}\right)=\lambda_{2}+\lambda_{3} \\
\frac{\partial}{\partial a_{2}}: & G^{\prime}\left(a_{2}\right)=\lambda_{2} .
\end{array}
$$

These first-order conditions relate $\left(a_{0}, a_{1}, a_{2}, \lambda_{1}, \lambda_{2}, \lambda_{3}\right)$; the equality constraints then give $\left(r_{0}, r_{1}, r_{2}\right)$ in terms of $\left(a_{0}, a_{1}, a_{2}\right)$. For this example, the first order conditions governing $a_{0}$ and $a_{1}$ are identical. There is a unique optimal value for the minimization, so we can conclude that it is achieved when $a_{0}=a_{1}$. This is a pattern that is followed for all $f$ : when the decoding rules for two symbols $i$ and $j$ are equal, i.e., when $f(i, z)=f(j, z) \forall z \in\{0,1\}$, then $a_{i}=a_{j}$. We can therefore combine symbols $i$ and $j$ to form a single symbol with transition probability $a_{i}$ and marginal probability $r_{i}+r_{j}$. The solution then has at most two symbols and four parameters, three of which can be fixed by the equality constraints. This reduces our optimization to a search over one free parameter as desired. 
The solution for other decoding functions $f$ is found using a similar approach, and we take advantage of the symmetry of the problem to reduce the number of decoding functions we must consider. From Table I and the following discussion, there are at most three decoding rules $f(w, \cdot)$ that need be considered for each symbol. The problem is symmetric in the three symbols, hence it is only the number of symbols using each decoding rule that is important in computing a solution. When all three symbols have the same decoding rule, then the optimal transition probabilities are the same for each and the three symbols can be combined into one. This one-symbol solution has no free parameters. When two symbols have the same decoding rule, the optimal solution uses two symbols and has only one free parameter. Finally, when all three symbols have different decoding rules, the first order conditions have a solution that we give in terms of $a_{0}$ as

$$
\left(a_{1}, a_{2}, \lambda_{1}, \lambda_{2}, \lambda_{3}\right)=\left(\frac{1}{2},\left(1-a_{0}\right),-q_{0} G^{\prime}\left(a_{0}\right), 0, G^{\prime}\left(a_{0}\right)\right)
$$

where $a_{0}$ itself is the solution to $G\left(a_{0}\right)=\left(a_{0}-q_{0}\right) G^{\prime}\left(a_{0}\right)$. We solve for $a_{0}$ numerically and obtain $r_{0}, r_{1}$, and $r_{2}$ from the equality constraints.

Considering now the case when some of the inequality constraints are made active (i.e., are made into equality constraints), we make the following observations.

- Assume the constraint (9) on $r_{w}, w \in\{0,1,2\}$ is active; that is, $r_{w}=0$. Then symbol $W=w$ is never used and the optimization need consider only solutions with at most two symbols.

- If all three of the constraints in (10) are active, then all parameters are uniquely determined. If two are active, we have only one free parameter.

From the above, either 1) we require only one or two symbols, 2) two or more of the $a_{w}$ are constrained, or 3) none of the constraints on the $r_{w}$ and at most one of the constraints on the $a_{w}$ are active. Since cases 1) and 2) both leave at most one free parameter, we can easily compute the optimal solution for each. The nontrivial solutions arising from case 3) are listed below.

The first is when $a_{0}=0$ and the distortion constraint is $D=r_{1} q_{0}+$ $r_{2}\left(1-a_{2}\right)$. From the first-order conditions, we obtain

$$
G\left(a_{1}\right)=H\left(q_{0}\right)+\left(a_{1}+q\right) G^{\prime}\left(a_{1}\right)-q_{0} G^{\prime}\left(a_{2}\right) .
$$

We search over $a_{2}$ and use (17) to find $a_{1}$ given $a_{2}$.

The second is when $a_{0}=1$ and the distortion constraint is $D=$ $r_{1} a_{1}+r_{2} q_{0}$. We search over $a_{2}$ and obtain $a_{1}$ numerically from

$$
G\left(a_{1}\right)=H\left(q_{0}\right)-G^{\prime}\left(a_{2}\right)+a_{1} G^{\prime}\left(a_{1}\right) .
$$

The above analysis reduces the search for the optimal $W$ to three solution classes:

1) solutions with only one or two symbols;

2) solutions with $a_{1}=\frac{1}{2}, a_{2}=1-a_{0}$, and $a_{0}$ found numerically;

3) solutions where exactly one of the boundary constraints on $a_{w}$ is active.

Numerical experiments suggest that the best solutions from class 3 never outperform the best solutions from classes 1 and 2 .

\section{APPENDIX B}

\section{DIFFERENTIABILITY OF $K\left(A_{u}\right)$}

To apply the first order optimality conditions in the Heegard and Berger problem, we need to ensure that $K\left(a_{u}\right)=R\left(a_{u}, q_{0}, D_{1}\right)-$ $H\left(a_{u}\right)$ is differentiable with respect to $a_{u}$ for $0<a_{u}<1$. Here, $R\left(a_{u}, q_{0}, D_{1}\right)$ is the binary MSI rate-distortion function, determined in
Section IV, when $p_{0}=a_{u}$. Since $H\left(a_{u}\right)$ is differentiable with respect to $a_{u}$ for $0<a_{u}<1$, it remains to ensure that $R\left(a_{u}, q_{0}, D_{1}\right)$ is also differentiable for $0<a_{u}<1$.

As we show in Lemma 4 below, $R\left(a_{u}, q_{0}, D_{1}\right)$ is a continuous function of $a_{u}$, differentiable at all but a finite number of points. In an arbitrarily small neighborhood around each of these points, we smooth the function to make it differentiable; we do so without changing the functional value by more than an arbitrarily small amount $\epsilon>0$. We substitute the smoothed (and differentiable) form of $R\left(a_{u}, q_{0}, D_{1}\right)$ for the original in the definition of $K\left(a_{u}\right)$. By doing so, we alter the function we are minimizing in the Heegard and Berger problem by at most $\epsilon$; the effect on the derived rate-distortion result of assuming differentiability of $K\left(a_{u}\right)$ is thus negligible.

Lemma 4: $R\left(p_{0}, q_{0}, D_{1}\right)$ is a continuous function of $p_{0}$, differentiable at all but a finite number of points.

Proof: $R\left(p_{0}, q_{0}, D_{1}\right)$ is given by (4) as

$$
\begin{aligned}
R & \left(p_{0}, q_{0}, D_{1}\right) \\
& =\min _{f \in \mathcal{F}} \min _{\mu(w \mid x) \in \mathcal{M}\left(p_{0}, q_{0}, D_{1}, f\right)}\left(-G\left(p_{0}\right)+\sum_{w=0}^{2} r_{w} G\left(a_{w}\right)\right) \\
& =-G\left(p_{0}\right)+\min _{f \in \mathcal{F} \mu(w \mid x) \in \mathcal{M}_{X \mid\{Z\}}\left(p_{0}, q_{0}, D_{1}, f\right)}\left(\sum_{w=0}^{2} r_{w} G\left(a_{w}\right)\right)
\end{aligned}
$$

where $\mathcal{M}_{X \mid\{Z\}}\left(p_{0}, q_{0}, D_{1}, f\right)$ is the set of all test channels describing an auxiliary random variable $W$ with $|\mathcal{W}| \leq 3$ such that $W \rightarrow X \rightarrow Z$ and $E d(X, f(W, Z)) \leq D_{1}$. Differentiability of $G\left(p_{0}\right)$ is shown in [2]; we concentrate on showing that the remaining term is differentiable.

For any $p_{0}$, the minimum over all test channels in the definition of $R\left(p_{0}, q_{0}, D_{1}, f\right)$ can be obtained by a test channel with $|\mathcal{W}|=3$. That no more than three symbols are needed is established in [2], and for any solution with fewer than three symbols, there always exists a corresponding three-symbol solution that yields the same minimum value. (For instance, if the two-symbol solution $\left(a_{0}, a_{1}, r_{0}, r_{1}\right)$ is optimal, then so is the three-symbol solution $\left(a_{0}^{\prime}, a_{1}^{\prime}, a_{2}^{\prime}, r_{0}^{\prime}, r_{1}^{\prime}, r_{2}^{\prime}\right)=$ $\left(a_{0}, a_{1}, a_{1}, r_{0}, \frac{r_{1}}{2}, \frac{r_{1}}{2}\right)$.) For each $f$, we partition $\mathcal{M}\left(p_{0}, q_{0}, D_{1}, f\right)$ into a set of interior test channels (all $a_{w} \in(0,1)$ ) and sets of different types of boundary test channels (having one or more $a_{w} \in\{0,1\}$ ). For each of these sets, we can derive a set of first-order conditions similar to those in (16). For all sets, we obtain the same result as we did for the conditions in (16): the first-order conditions uniquely determine the values of $a_{0}, a_{1}$, and $a_{2}$. These values are independent of $p_{0}$. The value of $p_{0}$ affects only how we determine $r_{0}, r_{1}$, and $r_{2}$ as functions of $a_{0}, a_{1}$, and $a_{2}$ by applying the equality constraints. Moreover, we can show that the functions specifying $r_{0}, r_{1}$, and $r_{2}$ are always linear functions of $p_{0}$, because the equality constraint (2) is a linear function of $p_{0}$. Since the objective function $\sum_{w=0}^{2} r_{w} G\left(a_{w}\right)$ in the minimization is also a linear function of $\left(r_{0}, r_{1}, r_{2}\right)$, this implies that for any of the sets, the minimal value of the objective function changes as a linear function of $p_{0}$.

We find

$$
\min _{f \in \mathcal{F} \mu(w \mid x) \in \mathcal{M}_{X \mid\{Z\}}\left(p_{0}, q_{0}, D_{1}, f\right)}\left(\sum_{w=0}^{2} r_{w} G\left(a_{w}\right)\right)
$$

as a function of $p_{0}$ by taking the minimum of the solutions yielded by the different sets for each $f$, followed by the minimum over all $f$. There are a finite number of functions to consider in the minima, and each is linear in $p_{0}$. The desired result follows from the observation that the minimum of a finite number $M$ of linear functions is continuous and is differentiable at all but at most $M-1$ points. 


\section{APPENDIX C \\ THE BINARY HB EXAMPLE}

In this Appendix, we outline the results of applying Lagrange multipliers to the minimization of (15) subject to the conditions in (11)-(14) and a distortion constraint.

There are only two possible decoding rules for each symbol: $f(u)=$ 0 or $f(u)=1$. When $f(u)=0$, then that symbol contributes an expected distortion of $r_{u} a_{u}$; when $f(u)=1$ it contributes an expected distortion of $r_{u}\left(1-a_{u}\right)$.

Consider first the case in which none of the inequality constraints is active. The application of Lagrange multipliers yields that $a_{u}=c_{1, f}$ for all $u$ such that $a_{u} \leq 1-a_{u}$, and $a_{u}=c_{2, f}$ otherwise. Since we can combine symbols with identical transition probabilities, then for any $f$ the optimal $U$ requires only two symbols. The two-symbol solution has four parameters, $\left(a_{0}, a_{1}, r_{0}, r_{1}\right)$. Three can be determined from the equality constraints, leaving one to search over. The evaluation of $R\left(a_{u}, q_{0}, D_{1}\right)$ also involves a search over one free parameter (as shown in the previous section), so that evaluating the optimal $U$ requires a search over two parameters.

Now consider the case when one or more of the inequality constraints are active. The inequality constraints on $r_{u}$ are of little interest since setting any particular $r_{u}$ to zero simply reduces the number of symbols by one. In applying the inequality constraints on the transition probabilities $a_{u}$, we first note that if $a_{i}=a_{j}=0$, or $a_{i}=a_{j}=1$ then symbols $i$ and $j$ can be combined into a single symbol. Therefore, there are only three cases of boundary solutions: one or more of the transition probabilities is zero, one or more is one, or some are zero and some are one. In all cases the boundary solution can be computed with a search over at most two parameters. We list below the three cases that require numerical solution of one or more parameters.

1) When $a_{0}=0$ and $D=a_{1} r_{1}+\left(1-a_{2}\right) r_{2}$, then we obtain $a_{1}$ and $a_{2}$ numerically in sequence from

$$
\begin{aligned}
& K\left(a_{1}\right)=a_{1} K^{\prime}\left(a_{1}\right) \\
& K\left(a_{2}\right)=\left(a_{2}-\frac{1}{2}\right) K^{\prime}\left(a_{2}\right)+\frac{1}{2} K^{\prime}\left(a_{1}\right) .
\end{aligned}
$$

2) When $a_{0}=1$ and $D=a_{1} r_{1}+\left(1-a_{2}\right) r_{2}$, then we obtain $a_{1}$ and $a_{2}$ numerically in sequence from

$$
\begin{aligned}
& K\left(a_{2}\right)=\left(a_{2}-1\right) K^{\prime}\left(a_{2}\right) \\
& K\left(a_{1}\right)=\left(a_{1}-\frac{1}{2}\right) K^{\prime}\left(a_{1}\right)-\frac{1}{2} K^{\prime}\left(a_{2}\right) .
\end{aligned}
$$

3) When $a_{0}=0, a_{1}=1$, and $D=r_{1}+a_{2} r_{2}+\left(1-a_{3}\right) r_{3}$, then we obtain $a_{2}$ and $a_{3}$ numerically from

$$
\begin{aligned}
& K\left(a_{2}\right)=a_{2} K^{\prime}\left(a_{2}\right) \\
& K\left(a_{3}\right)=\left(a_{3}-1\right) K^{\prime}\left(a_{3}\right) .
\end{aligned}
$$

Thus, when one or more of the inequality constraints is active, we can still evaluate the solution by searching over a total of two parameters.

\section{ACKNOWLEDGMENT}

The authors would like to thank the reviewers for their time and feedback, and they acknowledge Reviewer B for suggesting the shortened proof of Lemma 3 that appears in this work.

\section{REFERENCES}

[1] R. M. Gray, "A new class of lower bounds to information rates via conditional rate-distortion functions," IEEE Trans. Inf. Theory, vol. IT-19, no. 4, pp. 480-489, Jul. 1973.

[2] A. D. Wyner and J. Ziv, "The rate-distortion function for source coding with side information at the decoder," IEEE Trans. Inf. Theory, vol. IT-22, pp. 1-10, Jan. 1976.
[3] A. D. Wyner, "The rate-distortion function for source coding with side information at the decoder-ii: General sources," Inf. Contr., vol. 38, pp. 60-80, 1978.

[4] C. Heegard and T. Berger, "Rate distortion when side information may be absent," IEEE Trans. Inf. Theory, vol. IT-31, no. 6, pp. 727-734, Nov. 1985.

[5] K. J. Kerpez, "The rate-distortion function of a binary symmetric source when side-information may be absent," IEEE Trans. Inf. Theory, vol. IT-33, no. 3, pp. 448-452, May 1987.

[6] T. M. Cover and M. Chiang, "Duality between channel capacity and rate distortion with two-sided state information," IEEE Trans. Inf. Theory, vol. 48, no. 6, pp. 1629-1638, Jun. 2002.

[7] N. Merhav and S. Shamai (Shitz), "On joint source-channel coding for the Wyner-Ziv source and the Gel'fand-Pinsker channel," IEEE Trans. Inf. Theory, vol. 49, no. 11, pp. 2844-2855, Nov. 2003.

[8] R. M. Gray, "Conditional Rate-Distortion Theory," Stanford Electronic Labs., Stanford, CA, Tech. Rep. (6502-2), Oct. 1972.

[9] R. Zamir, "The rate loss in the Wyner-Ziv problem," IEEE Trans. Inf. Theory, vol. 42, no. 6, pp. 2073-2084, Nov. 1996.

[10] A. H. Kaspi, "Rate-distortion function when side-information may be present at the decoder," IEEE Trans. Inf. Theory, vol. 40, no. 6, pp. 2031-2034, Nov. 1994.

\section{Zero-Error Instantaneous Coding of Correlated Sources With Length Constraints Is NP-Complete}

\author{
Ying-On Yan, Member, IEEE, and Toby Berger, Fellow, IEEE
}

Abstract-It is well known that the Kraft inequality gives a necessary and sufficient condition on the codeword lengths of a zero-error instantaneous code for a single source. However, generalization for two correlated sources is nontrivial. We show that in the Slepian-Wolf configuration, even if one source is known at the decoder, designing a zero-error instantaneous code with given codeword lengths for the other source is NP-complete.

Index Terms-Correlated sources, Kraft inequality, NP-complete problem, prefix condition, rectangle packing, zero-error variable-length codes.

\section{INTRODUCTION}

Three decades have passed since Slepian and Wolf published their landmark paper on coding of correlated sources [1]. Nevertheless, most subsequent results on multiterminal source coding are concerned with block codes and a comprehensive theory of zero-error variable-length codes for multiple sources still needs to be developed. In this correspondence, we study the problem of generalizing a well-known result for variable-length codes, namely the Kraft inequality, for two correlated sources.

Let $X$ and $Y$ be discrete memoryless sources with alphabets $\mathcal{X}=$ $\left\{0,1,2, \ldots, n_{X}-1\right\}$ and $\mathcal{Y}=\left\{0,1,2, \ldots, n_{Y}-1\right\}$, respectively.

Manuscript received December 17, 2002; revised December 24, 2005. The material in this correspondence was presented in part at the 2002 IEEE International Symposium on Information Theory, Lausanne, Switzerland, June 2002.

Y.-O. Yan is with The MathWorks, Inc., Natick, MA 01760 USA (e-mail: yoyan@ieee.org).

T. Berger was with the School of Electrical and Computer Engineering, Cornell University, Ithaca, NY 14853 USA. He is now with the Department of Electrical and Computer Engineering, University of Virginia, Charlottesville, VA 22903 USA (e-mail: tb6n@ virginia.edu).

Communicated by S. A. Savari, Associate Editor for Source Coding.

Digital Object Identifier 10.1109/TIT.2006.871039 